\title{
Automatic Optimization of Chirp Setting Parameters in Medical Ultrasound Contrast Imaging
}

\author{
Amira Zaylaa ${ }^{1,2,3}$, Sébastien Ménigot ${ }^{1,2,4}$, Jamal Charara ${ }^{3}$ and Jean-Marc Girault ${ }^{1,2}$ \\ 1. Department of Medical Biophysics and Imaging, Université François Rabelais de Tours UMR-S930 and ERL3106, B.P 3223, \\ Tours, France \\ 2. INSERM, U930, Tours, France \\ 3. Department of Physics and Electronics, Lebanese University, Hadath Campus, B.P. 5, Beirut, Lebanon \\ 4. IUT Ville d'Avray, Université Paris Ouest Nanterre La Defense, B.P. 105, Avray, France
}

Received: February 16, 2013 / Accepted: April 09, 2013 / Published: June 30, 2013.

\begin{abstract}
Medical ultrasound contrast imaging is a powerful modality undergoing successive developments in the last decade to date. Lately, pulse inversion has been used in both ultrasound tissue harmonic and contrast imaging. However, there was a tradeoff between resolution and penetration. Chirp excitations partially solved the tradeoff, but the chirp setting parameters were not optimized. The present work proposes for the first time combining chirp inversion with ultrasound contrast imaging, with the motivation to improve the contrast, by automatically optimizing the setting parameters of chirp excitation, it is thus an optimal command problem. Linear chirps, $5 \mu \mathrm{m}$ diameter microbubbles and gradient ascent algorithm were simulated to optimize the chirp setting parameters. Simulations exhibited a gain of $5 \mathrm{~dB}$ by automatic optimization of chirp inversion relative to pulse inversion. The automatic optimization process was quite fast. Combining chirp inversion with ultrasound contrast imaging led to a maximum backscattered power permitting high contrast outcomes and optimum parameters.
\end{abstract}

Key words: Medical ultrasound contrast imaging, chirp inversion, chirp setting parameters, automatic technique, optimal command.

\section{Introduction}

Ultrasound contrast imaging is an inevitable modality in medicine. It has significantly improved medical diagnosis by ameliorating the contrast after injecting a contrast agent into the patient's vascular system [1]. Several methods have been proposed in literature to improve the CTR (contrast-to-tissue ratio). These methods were classified into two major groups. The first group (i) was based on continuous encoding such as HCI (harmonic chirp imaging) [2, 3]. HCI has improved resolution and increased the SNR (signal-to-noise ratio) of images. It was also capable

Corresponding authors: Amira Zaylaa, Ph.D. student, research fields: signal processing and ultrasound imaging. E-mail: amira.zaylaa@inserm.fr.

Jean-Marc Girault, Ph.D., research fields: signal and image processing and medical instrumentation. E-mail: jmgrault@univ-tours.fr. of solving the trade-off between resolution and penetration $[4,5]$. The second group (ii) was based on discrete encoding imaging methods such as pulse inversion [6], contrast pulse sequence [7] and pulse subtraction [8]. These methods were capable of efficiently extracting the harmonic frequency components out of the spectrum of backscattered signals even if any overlap existed in this spectrum.

Few years later, in order to benefit from the two aforementioned groups that contributed to the enhancement of the CTR, several scientists have proposed to merge some techniques together. For instance, chirp reversal has been proposed in harmonic contrast imaging [9] and chirp inversion in tissue harmonic imaging [10].

In this paper, the authors have proposed for the first time to merge chirp inversion with harmonic 
ultrasound contrast imaging. The reason behind this was to benefit from the advantage of not only each approach alone but also their fusion. However, the contribution was not limited to this combination. Indeed, as it has been shown recently the necessity of setting chirp frequency parameters in an optimal way through a theoretical approach [11] and more recently, the possibility of automatically optimizing transmitted pulse parameters, without using apriori information of the medium perfused with microbubbles [12], authors proposed to set automatically and in an optimal way the chirp setting parameters in chirp inversion.

Besides, by automatically seeking the best power backscattered by microbubbles, the authors hope to increase the quality of harmonic ultrasound images and thus improve the medical diagnosis.

To advocate the feasibility and powerfulness of the novel proposed approach, the authors will compare, through simulations, the performances of the method to well-known pulse inversion imaging chosen as a reference method.

\section{Materials}

In this section, the coded ultrasound contrast imaging system is presented (Fig. 1). Such system is used to image the perfused medium (the liver for instance). To achieve this task, physicians inject microbubbles, the constituents of ultrasound contrast

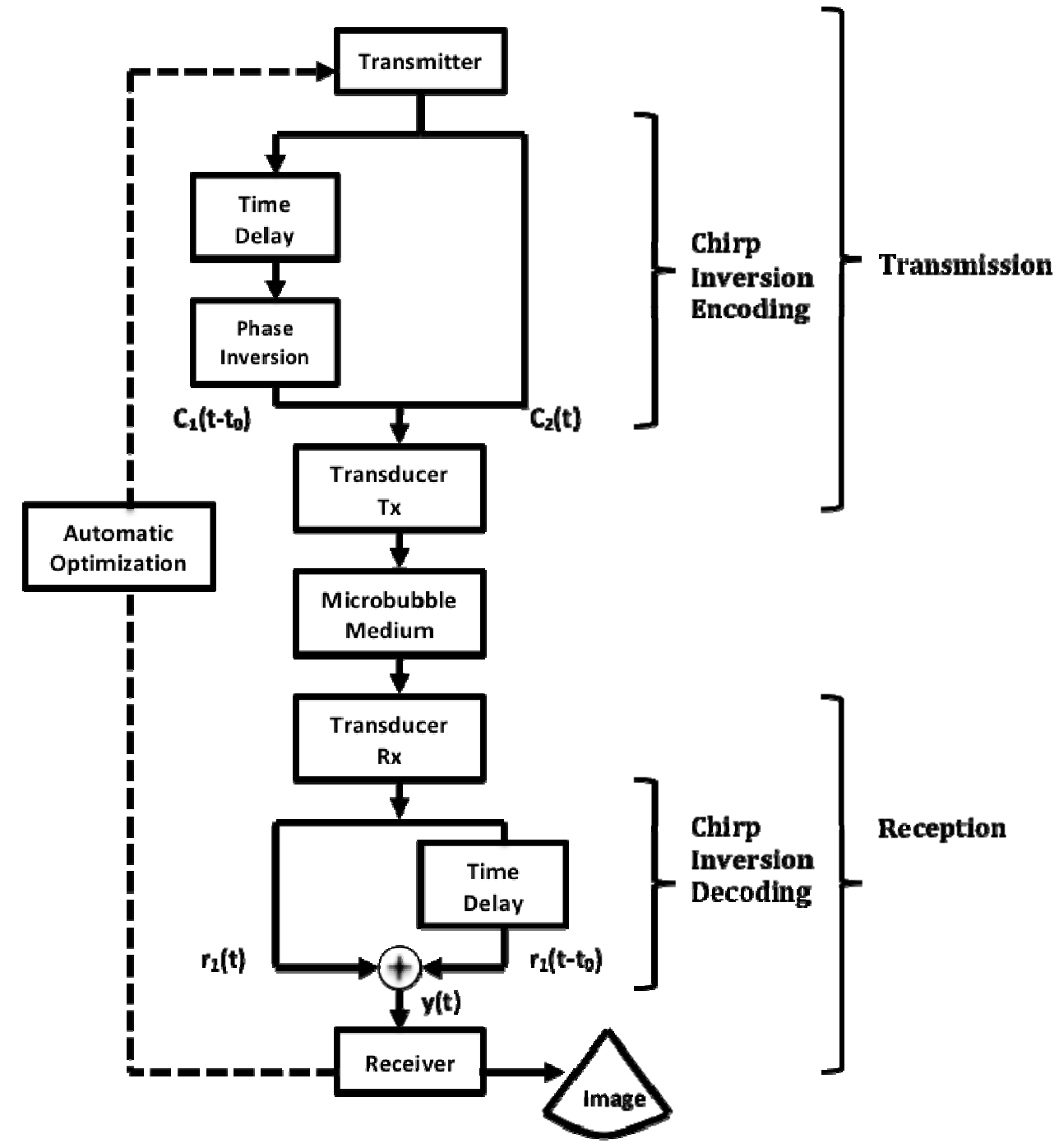

Fig. 1 Block diagram of chirp inversion in ultrasound contrast imaging. 
agent (Subsection 2.3). At the same time, ultrasound chirp excitation (Subsection 2.1), which is transmitted by a transducer to the explored medium, is encoded and simulated (Subsection 2.2). When the ultrasound waves interact with the medium perfused with microbubbles, echoes are reflected and backscattered towards the receiver (Subsection 2.4). At the receiver, the electrical signals are decoded and used to construct the image.

\subsection{Transmitter, Transmitting Transducer and Chirp Excitations}

As already mentioned, a transducer was used to transmit chirp excitations. During transmission, these chirps were filtered by the passband filters of the transmitting transducer Tx (Fig. 1). The bandwidth of the simulated transmitting transducer was $74 \%$ at -3 $\mathrm{dB}$ and central frequency $2.25 \mathrm{MHz}$. Chirp excitations are signals possessing a dynamic (instantaneous) frequency. A chirp $C_{j}(t)$ is said to be linear when its dynamic frequency is linear (Eq. (1)) [10]:

$$
\left\{\begin{array}{c}
C_{j}(t)=A_{0} \exp \left(-\frac{\left(t-t_{0}\right)^{2}}{2 \sigma^{2}}\right) \cdot \cos \left(\theta_{i}(t)\right) \\
\theta_{i}(t)=2 \pi\left(f_{i}(t)\right) t+\varphi_{j} \\
f_{i}(t)=f+\beta t
\end{array}\right.
$$

where $A_{0}$ is the normalized driving pressure of the chirp; $t_{0}$ : time delay; $t$ : time; $f$ : starting frequency or first setting parameter of chirp; $\beta$ : frequency modulation index or second setting parameter; $f_{i}$ : instantaneous frequency; $\varphi$ : angular phase; $\theta_{i}$ : the instantaneous phase of the chirp; and $\sigma$ : width of the Gaussian's envelope, which was chosen through the simulation as proposed by Wilhjelm [13] to be 3. Note that the well-known pulse definition can be obtained from Eq. (1) by setting $\beta=0$. Chirp excitations were then encoded.

\subsection{Chirp Inversion Encoding}

Chirp inversion encoding simulated with Matlab (Mathworks, Natick, MA, USA) is the process of transmitting two $180^{\circ}$ out of phase chirps sequentially into the medium perfused with microbubbles. Chirp echoes reflected by the contrast agent are then summed up such that echoes at the starting frequency $f$ cancel out while those at the harmonic frequencies show up $[6,10,14]$. Chirp inversion encoding was illustrated by the two branches following transmission in Fig. 1. The first emitted chirp $C_{1}(t)$ was fed into a time-delay block and then a phase inverse block to get $C_{1}\left(t-t_{0}\right)$ with $\varphi_{1}=\pi$. The second chirp was $C_{2}(t)$ with $\varphi_{2}=0$. Then $C_{1}\left(t-t_{0}\right)$ and $C_{2}(t)$ were filtered and fed into the medium of microbubbles.

\subsection{Microbubble Model}

Simulated medium consisted of micrometer-sized bubbles. For simplicity, and without loss of generality, in order to simulate the mean behavior of a microbubble cloud, it was assumed that the response (non-linear vibration) of a cloud of $N$ microbubbles was $N$ times the response of a single microbubble possessing the mean properties.

Bubblesim software [15] was used in this work, in order to account for the microbubble's response when exposed to a megahertz-frequency ultrasound chirp. It is based on simulating the response by digitally solving modified Rayleigh-Plesset model (Eq. (2)).

$$
\begin{gathered}
\ddot{R}(t) \dot{R}(t)+\frac{3}{2} \dot{R}(t)^{2}+\frac{p_{0}+p_{i}(t)+p_{L}(t)}{\rho_{L}} \\
-\frac{p_{L}(t)}{p_{L} c_{L}} R(t)=0
\end{gathered}
$$

where $R$ is the instantaneous radius of microbubbles, $\dot{R}$ and $\ddot{R}$ are first and second derivative respectively, $p_{0}$ : static pressure; $p_{i}$ : instantaneous acoustic pressure; $p_{L}$ : liquid pressure on the surface of the micro-bubbles; $\rho_{L}$ : liquid density; and $c_{L}$ : velocity of sound in the liquid. Eq. (2) was based on modeling microbubbles by air-filled particles where the surface layer resembles that of an elastic solid.

SonoVue [16] contrast agent was simulated according to experiments in literature. The mean diameter of simulated SonoVue (Bracco Research SpA, Geneva, Switzerland) microbubbles was $5 \mu \mathrm{m}$ [16] and the shell thickness $d_{S e} 1 \mathrm{~nm}$ [17]. The shell 
shear modulus $G_{S}$ was $46 \mathrm{MPa}[15]$ and the shear viscosity $1 \mathrm{~Pa} \cdot \mathrm{s}$.

\subsection{Receiving Transducer, Chirp Inversion Decoding and Receiver}

The first element of the reception stage (Fig. 1) was the transducer $R x$, the bandwidth of $R x$ was $63 \%$ at -3 $\mathrm{dB}$ and central frequency 3.5 MHz. For illustration, the transducer's effect was taken into consideration in transmission and reception, such that, its bandwidth was $160 \%$ at $-3 \mathrm{~dB}$ and central frequency $3 \mathrm{MHz}$. The received echoes from the same transducer were used for constructing the ultrasound image. The backscattered signals were decoded (Eq. (3)) and a time delay was imposed on the second backscattered chirp (Fig. 1).

$$
\left\{\begin{array}{l}
r_{1}(t)=e \operatorname{cho}\left\{C_{1}\left(t-t_{0}\right)\right\} \\
r_{2}\left(t-t_{0}\right)=e \operatorname{echo}\left\{C_{2}(t)\right\}
\end{array}\right.
$$

Then the backscattered chirp $y(t)$ was computed (Eq. (4)).

$$
y(t)=r_{1}(t)+r_{2}\left(t-t_{0}\right)
$$

The former phase is called feed forward phase.

\section{Method}

In this section, the automatic optimization of the chirp setting parameters that enabled to optimize the power in the ultrasound image was presented, thanks to the feedback line (dashed line in Fig. 1).

\subsection{Automatic Optimization Technique}

Automatic optimization was based on gradient ascent technique inspired from adaptive filtering. It is an iterative process that tracks the maximum of a cost function $J$ [18] being the backscattered power:

$$
J\left(\phi_{k}\right)=\int y\left(t, \phi_{k}\right)^{2} d t
$$

where $\phi_{k}=\left(f_{k}, \beta_{k}\right)^{T}$ is the vector of the setting parameters to be optimized at the $k^{\text {th }}$ iteration, $T$ : the transpose; $f_{k}$ : the starting frequency; $\beta_{k}$ : the modulation index; and $y\left(t, \phi_{k}\right)$ : the backscattered chirp of inversion decoding. The automatic algorithm was implemented to find the maximum of $J\left(\phi_{k}\right)$ at $f^{*}$ and $\beta^{*}$ according to the following equation:

$$
\phi_{k}{ }^{*}=\underset{\phi}{\operatorname{argmax}}\left(J\left(\phi_{k}\right)\right)
$$

The automatic algorithm iteratively generates the optimum values of $f^{*}$ and $\beta^{*}$ from $J\left(\phi_{k}\right)$. The algorithm was summarized in the following points:

- Three initial values of $(f, \beta)$ parameters thus far have been predefined. The algorithm launched in the forward stage for the first three iterations $k=1,2,3$;

- The output of chirp inversion processing (i.e. forward process) was fed into the optimization feedback after the third iteration, i.e. $\{k \geq 4\}$;

- The cost function was measured as in Eq. (5);

- The iterative equation (Eq. (6)) was then implemented to compute steps proportional to the positive of the gradient of $J$ at the current point. This step was responsible of setting the maximum power that could have been attained. It was equivalent to:

$$
\phi_{k+1}=\phi_{k}+\mu\left(\nabla_{\phi_{k}} J\left(\phi_{k}\right)\right)
$$

where $\mu$ was the speed of convergence of $f^{*}$ and $\beta^{*}$ to the optimum value, it was initialized absolutely to 2 . The choice of 2 was settled on after testing $0.1 \leq \mu \leq 3$;

- The gradient was approximated by the discrete gradient (Eq. (8)) as there was no analytical expression that describes the variation of the power as a function of the frequency parameters given below:

$$
\nabla_{\phi_{k}}\left(J\left(\phi_{k}\right)\right)=\frac{J\left(\phi_{k}\right)-J\left(\phi_{k-1}\right)}{\phi_{k}-\phi_{k-1}}
$$

The power of $y\left(t, \phi_{k}\right)$ converged and $\left(f^{*}, \beta^{*}\right)$ were automatically obtained.

\subsection{Gain}

The power gain $G_{d B}$ was defined as the ratio of the automatically optimized chirp inversion system (i.e. the block diagram with the feedback stage (Fig. 1) to the pulse inversion imaging [6], i.e. for $\beta=0$ (as mentioned in Subsection 2.1). In pulse inversion, the frequency parameters were set to $f_{\text {ref }}=2.25 \mathrm{MHz}$ and $\beta=0 \mathrm{MHz} / \mathrm{s}$ [19]. Therefore, the gain $G_{d B}$ was obtained by the following equation:

$$
G_{d B}=10 \cdot \log _{10}\left(\frac{J\left(f^{*}, \beta^{*}\right)}{J\left(f_{\text {ref }}, 0\right)}\right)
$$

\section{Simulation Results and Discussion}

Simulations were carried out on an ultrasound 
contrast agent composed of $5 \mu$ m-diameter bubbles. A linear chirp of driving pressure $100 \mathrm{kPa}$ with three initial predefined central transmitted frequencies 1.5, 3 and $3.5 \mathrm{MHz}$ as proposed in Ref. [12] and initial second frequency parameter assigned the values 0.2 , 0.4 and $0.6 \mathrm{MHz} / \mathrm{s}$ were tested using the automatic algorithm.

Fig. 2 represented the power image of the backscattered chirp $y(t)$, coded in colors, as a function of $f$ and $\beta$. The chirp parameters $f$ and $\beta$ were ranging between $0.5 \mathrm{MHz}$ and $6.5 \mathrm{MHz}$ and $-2 \mathrm{MHz} / \mathrm{s}$ and $2 \mathrm{MHz} / \mathrm{s}$, respectively. The power was computed empirically, i.e., point-by-point by (Eq. (5)) in logarithmic unit. The power values were associated with colormap jet, such that, points exhibiting high power were associated a dark red color and those exhibiting lowest power outcomes were associated dark blue color. This power was considered as our cost function.

From Fig. 2, it was obvious that the maximum of the cost function was obtained for $\beta$ different from zero and for $f$ different from $f_{\text {ref }}$. This empirically demonstrated the necessity of using chirp excitations and thus seeking the optimal value of the two setting parameters $\beta$ and $f$ that maximizes the cost function. Nevertheless, instead of empirically testing all the $(f, \beta)$ couples, we desired to achieve this optimally and automatically using a gradient ascent method.

Since the image seemed to be symmetric with respect to the line $\beta=0$, it was sufficient to merely study the cost function for $\beta>0$. So we have super imposed on (Fig. 2) the trajectory of the automatic technique that led to the optimal values. The bold black line elaborated the novel automatic optimization path and the circles indicated the number of iterations that was 7 , after which the algorithm converged to the optimum setting parameters. Note that $f_{\text {ref }}$ denotes the frequency value of the reference method, which is pulse inversion as mentioned in Section 1.

Fig. 3 represented the variation of the power of the backscattered chirp $y(t)$ as a function of the number of iterations for chirps of driving pressure $100 \mathrm{kPa}$. In the first iteration, the power of chirp was -32.1 logarithmic power units, then as the number of iterations increased the power increased gradually, then decreased at the fourth iteration. Afterwards it increased until it reached its maximum of -30.8 logarithmic power units at the seventh iteration and attained a plateau until the fiftieth iteration. This variation in the power values proves the sensitivity of the cost function $J$ to the choice of $(f, \beta)$ couples. Note that the power obtained by pulse inversion was illustrated by the horizontal dashed line (Fig. 3) and the power difference was approximately $5 \mathrm{~dB}$.

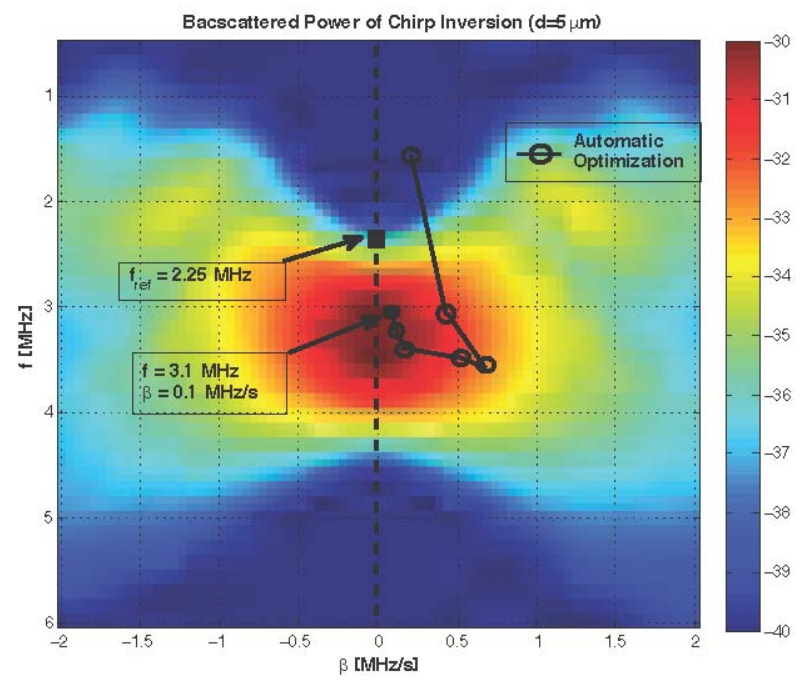

Fig. 2 The backscattered power (coded in colors) of the linear chirp $y(t)$ of driving pressure $100 \mathrm{kPa}$ as a function of $f$ and $\beta$ with the automatic optimization trace.

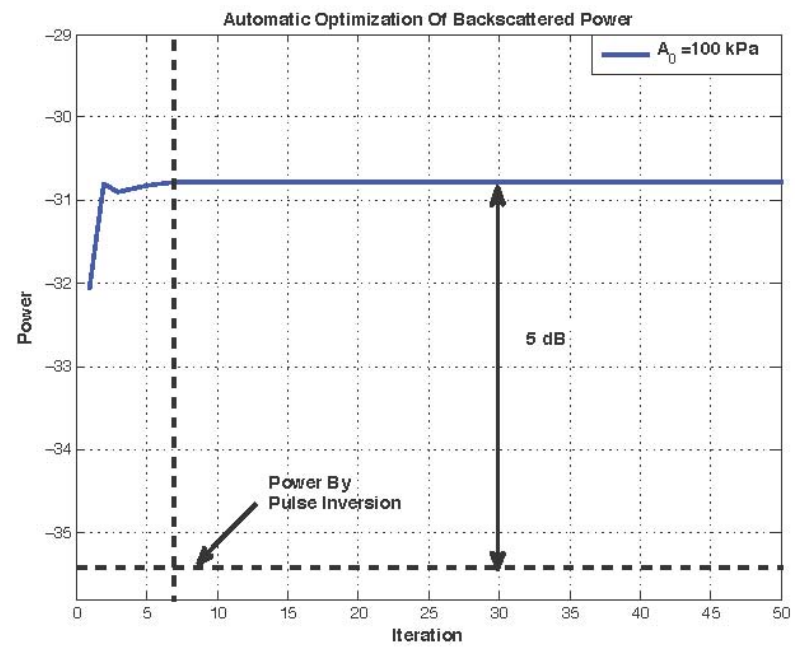

Fig. 3 Automatic optimization of the backscattered power of chirp $y(t)$ with initial driving pressure $100 \mathrm{kPa}$. 


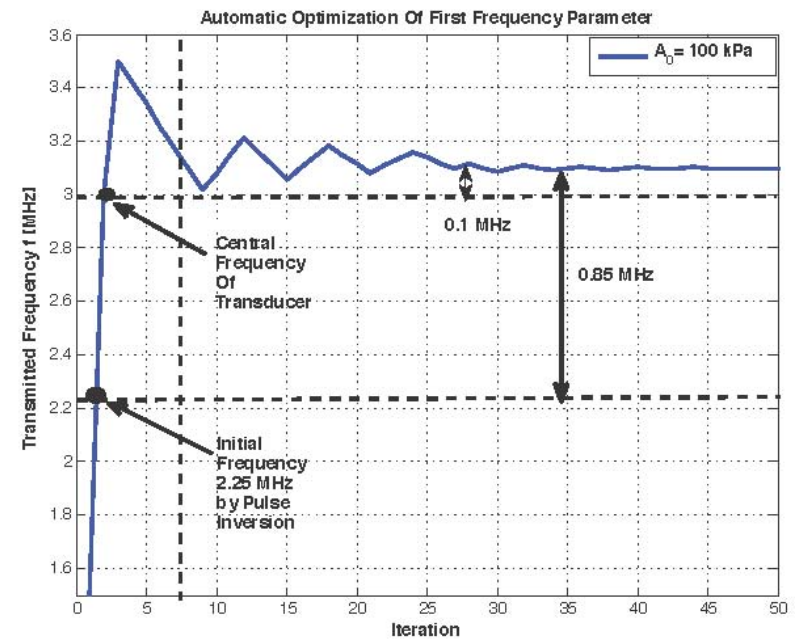

Fig. 4 Automatic optimization of the first chirp parameter $f$ for initial driving pressure $100 \mathrm{kPa}$.

Fig. 4 manifested the automatic optimization of $f$ as a function of the number of iterations. The optimum value of the first setting parameter was attained at the point of convergence of the power, i.e., at the seventh iteration. The algorithm converged to $3.1 \mathrm{MHz}$. This implied that the optimal transmitted frequency was 3.1 $\mathrm{MHz}$ and was different from the reference frequency $\mathrm{f}_{\text {ref }}=2.25 \mathrm{MHz}$ in pulse inversion. The optimal frequency was $0.1 \mathrm{MHz}$ above the central frequency of the transducer and $0.85 \mathrm{MHz}$ above the reference frequency 2.25 $\mathrm{MHz}$ in pulse inversion [12], regardless of the initial driving pressure level $\mathrm{A}_{0}$.

Fig. 5 exhibited the variation of the second frequency chirp setting parameter $\beta$ as a function of the number of iterations. The optimum value was 0.1 $\mathrm{MHz} / \mathrm{s}$, selected after the seventh iteration where the power converged to its maximum value.

Table 1 represented the optimal setting parameters in automatic chirp inversion in addition to the gain (Eq. (9)). The novel automatic technique of chirp inversion achieved a gain of $5 \mathrm{~dB}$ as compared to the existing pulse inversion technique.

To sum up, chirp's frequency setting parameters, transmitted frequency $f$ and frequency modulation index $\beta$, were optimized and thought to improve the backscattered power. Results showed that the proposed optimization method does not require apriori

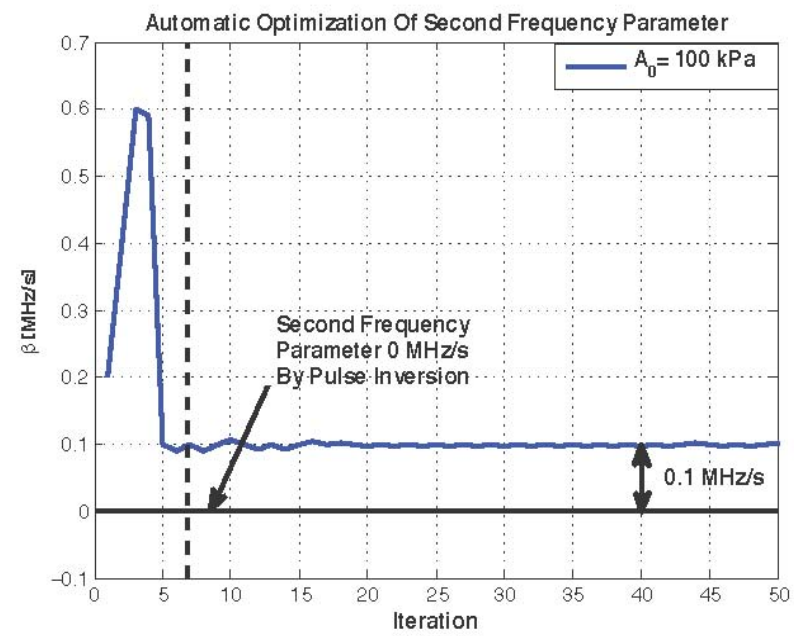

Fig. 5 Automatic optimization of the second chirp setting parameter $\beta$ for initial driving pressure $100 \mathrm{kPa}$.

Table 1 Automatic optimization setting parameters and gain.

\begin{tabular}{|c|c|c|}
\hline \multicolumn{2}{|c|}{ Setting parameters } & \\
\hline$f^{*}(\mathrm{MHz})$ & $\beta^{*}(\mathrm{MHz} / \mathrm{s})$ & - Gain (dB) \\
\hline 3.1 & 0.1 & 5 \\
\hline
\end{tabular}

knowledge of the microbubble, since it requires merely the cost-function. Simulations revealed that optimization feedback in chirp inversion was crucial. A $5 \mathrm{~dB}$ gain was achieved by the automatic feedback as compared to pulse inversion where $\beta=0$. The speed of convergence of the algorithm was appreciated such that it was attained in seven iterations knowing that there were three measurements required per iteration.

If the cost function was neither convex nor concave, the initial conditions of the frequency parameters should be carefully chosen. This could be fulfilled by testing various points to meet the demand. Otherwise, additional optimization technique capable of selecting the proper initial conditions should be considered.

The major practical advantage of the proposed technique is that neither the technician nor clinician is required to tune his transducer to any frequency value. Indeed, the technique will automatically adapt itself and select the optimum setting parameters that maximize the backscattered power from the patient. The enhancement of the resolution and SNR guaranteed by the use of chirp excitations was 
associated with iterative power increase; this might improve the CTR of contrast ultrasound images during the whole examination even if different kinds of variations occurred.

\section{Conclusion}

Chirp inversion in ultrasound contrast imaging accompanied by automatic optimization technique enhances the backscattered power. Since the technique is not limited to linear chirps, other chirp types could be investigated. However, experimental verification of the simulation is needed.

\section{Acknowledgments}

The authors are grateful to the National Council for Scientific Research in Lebanon, Lebanese University and Imaging and Brain Laboratory INSERM U930, François Rabelais University of Tours in France for supporting this work.

\section{References}

[1] P.J. Frinking, A. Bouakaz, J. Kirkhorn, F.J. Ten Cate, N. de Jong, Ultrasound contrast imaging: Current and new potential methods, Ultrasound in Medicine and Biology 26 (2000) 965-975.

[2] J.M.G. Borsboom, C.T. Chin, N. de Jong, Nonlinear coded excitation method for ultrasound contrast imaging, Ultrasound in Medicine and Biology 29 (2003) 277-287.

[3] J.M.G. Borsboom, C.T. Chin, A. Bouakaz, M. Versluis, N. de Jong, Harmonic chirp imaging method for ultrasound contrast agent, IEEE Transaction on Ultrasonic, Ferroelectric and Frequency Control 52 (2005) 241-249.

[4] T. Misaridis, J.A. Jensen, U se of modulated excitation signals in medical ultrasound, Part I: Basic concepts and expected benefits, IEEE Transaction on Ultrasonic, Ferroelectric and Frequency Control 52 (2005) 177-191.

[5] R.Y. Chiao, X. Hao, Coded excitation for diagnostic ultrasound: A system developer's perspective, IEEE Transaction on Ultrasonic, Ferroelectric and Frequency Control 52 (2005) 160-170.

[6] D.H. Simpson, C.T. Chin, P.N. Burns, Pulse inversion Doppler: A new method for detecting nonlinear echoes from microbubble contrast agents, IEEE Transaction on
Ultrasonic, Ferroelectric and Frequency Control 46 (1999) 372-382.

[7] A.G. Brock-fisher, D.M. Poland, G.P. Rafter, Means for increasing sensitivity in non-linear ultrasound imaging systems, US Patent 5577 505, 1996.

[8] J.M.G. Brosboom, A. Bouakaz, N. de Jong, Pulse subtraction time delay imaging method for ultrasound contrast agent detection, IEEE Transaction on Ultrasonic, Ferroelectric and Frequency Control 56 (2009) 1151-1158.

[9] A. Bouakaz, Chirp reversal ultrasound contrast imaging, EPO Patent EP1739455, 2007.

[10] J. Song, J.H. Chang, T.K. Song, Y. Yoo, Coded tissue harmonic imaging with nonlinear chirp signals, Ultrasonics 51 (2011) 516-521.

[11] E. Barlow, A.J. Mulhlland, A. Gachagan, A. Nordon, A theoretical investigation of chirp insonification of ultrasound contrast agents, Ultrasonics 51 (2011) 725-733.

[12] S. Menigot, J.M. Girault, I. Voicu, A. Novell, Optimization of contrast to tissue ratio by frequency adaptation in pulse inversion imaging, IEEE Transactions on Ultrasonics, Ferroelectrics and Frequency Control 59 (2012) 2431-2438.

[13] J.E. Wilhjelm, Bandwidth expressions of Gaussian weighted chirp, Electronics Letters 29 (1993) 2161-2162.

[14] M. Pasovic, M. Danilouchkine, T. Faez, P.L.M.J. van Neer, C. Cachard, A.F.W. van der Steen, et al., Second harmonic inversion for ultrasound contrast harmonic imaging, Physics in Medicine and Biology 56 (2011) 3163-3180.

[15] L. Hoff, Acoustic Characterization of Contrast Agents for Medical Ultrasound Imaging, Kluwer Academic, Boston, USA, 2001, pp. 158-160.

[16] C. Greis, Technology Overview: SonoVue (Bracco, Milan), European Radiology 14 (2004) 11-15.

[17] K. Chetty, C.A. Sennoga, J.V. Hainal, R.J. Eckersley, E. Stride, P1F-4 high speed optical observations and simulation results of lipid based microbubbles at low insonation pressures, in: Proceeding IEEE, Ultrasonics Symposium, 2006, pp. 1354-1357.

[18] R. Fletcher, Conjugate direction methods, in: W. Murray (Ed.), Numerical Methods for Unconstrained Optimization, Academic Press, New York, 1972.

[19] A. Zaylaa, S. Menigot, J.M. Girault, J. Charara, Empirical optimization of frequency parameters in chirp inversion imaging, Proceeding Acoustics 2012, Nantes, France, 273 (2012) 2889-2893. 